\title{
Major Risk Factors Contributing to Split Thickness Skin Graft Failure
}

\author{
Jon D Turissini ${ }^{1}$, Tammer Elmarsafi ${ }^{1}$, Karen K Evans ${ }^{1}$, Paul J Kim ${ }^{1}$ \\ ${ }^{1}$ Georgetown University Medical Center \\ Keywords: skin graft \\ https://doi.org/10.52504/001c.7755
}

\section{Georgetown Medical Review}

Vol. 3, Issue 1, 2019

A retrospective review was done for each wound $(n=223)$ in all patients $(n=$ 191) who underwent Split Thickness Skin Graft (STSG) placement in the Wound Division at Georgetown University Hospital from January 2014 to March 2017 in order to determine the factors that significantly affect STSG take. In doing so, these factors that prove to significantly affect STSG take can be used to predict the possibility of graft failure, and, thus, determine if additional measures must be taken in order to improve the success of the skin graft. Patient medical records were examined for patient demographics, comorbidities, wound parameters, wound bed prep, post-operative dressing, 30 day graft outcomes, and 60 day graft outcomes. Statistical analysis was performed to determine the significance of each factor, and further analysis was done to determine the association and risk of the statistically significant factors. Statistical analysis showed a significant association between Negative Pressure Wound Therapy (NPWT) for wound bed dressing after STSG placement and successful STSG outcome compared to use of bolster only for the post-surgical wound $\left(\chi^{2}=4.66\right.$, $\mathrm{p}=0.0308)$. The odds of STSG failure in patients who underwent NPWT were approximately $80 \%$ less than those who had bolster dressing used for their postsurgical dressing $(\mathrm{OR}=0.203)$. These results indicate that NPWT after skin graft placement yields a greater success rate for split-thickness skin grafts than conventional bolster dressing. In terms of comorbidities, there was also a significant association between congestive heart failure (CHF) and STSG failure $\left(\chi^{2}=4.12, p=0.0422\right)$. Patients with CHF were approximately 2.55 times more likely to have their STSG fail $(\mathrm{OR}=2.55)$, indicating that $\mathrm{CHF}$ is a good predictor of split-thickness skin graft failure. It was also found that bacterial presence and STSG failure also showed an association $\left(\chi^{2}=4.66, p=0.0308\right)$, in which patients with bacterial presence on the wound prior to debridement were approximately 2.89 times more likely to have STSG failure $(\mathrm{OR}=2.89)$. Although bacterial presence prior to debridement showed an association with STSG failure, bacterial presence after debridement just prior to STSG placement did not show a significant correlation with STSG failure $\left[\mathrm{n}_{\mathrm{f}}=52(73.2 \%)\right.$ versus $\left.\mathrm{n}_{\mathrm{s}}=95(62.5 \%),(\mathrm{p}=0.1150)\right]$. These results suggest that bacterial presence may also be a good predictor of graft failure, however it is possibly the strain of bacteria, not the presence of bacteria that predominantly affects skin graft take. In order to elucidate the role that bacteria plays in the success of STSG take, further experimental analysis is warranted.

\section{Introduction}

Skin is the not only the largest organ in the body, but is also the first line of defense from the external environment, pivotal in thermoregulation, and involved in metabolic function, and thus its integrity is of the utmost importance. Large or non-healing insults to this integumentary layer leaves the body especially susceptible to infection and further insult, and failure to rectify the wound can lead to disastrous outcomes. Large wounds often involve extensive parenchymal cell death and extracellular framework destruction, and continued inflammation of these wounds causes further destruction, hindering the body's natural regenerative properties. Split thickness skin grafts 


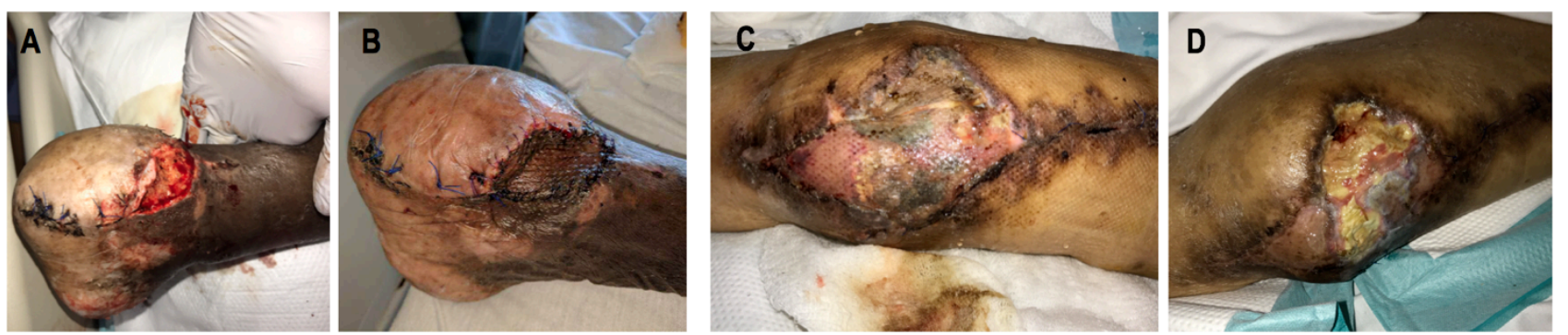

Fig. 1(A-D). A) Anterior ankle wound status post Chopart's amputation. B) Anterior ankle wound post-op with successful take of split-thickness skin graft (STSG). C) Large knee wound status post STSG placement. D) Same knee wound post-op with 40\% STSG take, indicating STSG failure.

Figure 1

(STSG), autografts that include the entire epidermal and a portion of the dermal layer, are the gold standard used to cover and induce reparation of a variety of full thickness wounds from burns, traumatic injury, or non-healing diabetic ulcerations. The graft serves as a reservoir for cells necessary for wound repair, as well as structural and cell signaling proteins and growth factors. The intent of these graft components is to induce neovascularization and granulation tissue formation, followed by collagen deposition, extracellular matrix remodeling, and maturation, in order to speed the healing and repair processes of the surrounding skin and restore the integrity and function of this protective organ (Figure 1A-B). Lower extremity wounds are particularly susceptible to chronic wound formation due to their distal location along the arterial vascular system, which predisposes them to ischemia.

Though placing a STSG is a relatively non-invasive and simple procedure, the grafts are often prone to failure. Once the graft is harvested, it no longer receives the nutrients and oxygen from its host blood supply. If the graft is unsuccessful in adhering to the wound site and revascularizing via angiogenesis, or any factors impede this process, the graft risks failure (Figure 1C-D). Patient demographics, comorbidities, wound parameters, wound bed preparation, as well as the surgical dressing used after STSG placement are all factors that may affect the final take of the graft, however the most common cause of skin graft failure has been found to be hematoma formation, which prevents efficient adherence and revascularization of the skin graft. ${ }^{1}$ Other post-operative complications, such as seroma formation and infection, can also greatly affect the graft outcome. Despite the ubiquitous use of skin grafting, there has been a dearth of large studies assessing all of the major risk factors contributing to the outcomes of skin graft failure. The purpose of this retrospective study is to investigate the aforementioned factors and to examine the 30 and 60 day outcomes of the STSGs on wounds in patients who required their placement in order to assess the association between these factors and STSG failure. 


\section{Methods}

After securing Institutional Review Board approval, a retrospective review was done for lower extremity wounds $(n=223)$ in all patients $(n=191)$ who underwent STSG placement in the Wound Division at Georgetown University Hospital from January 2014 to March 2017. Patient medical records were examined for patient age, sex, diabetes mellitus (DM), hypertension, hyperlipidemia, congestive heart failure (CHF), renal failure, peripheral arterial disease (PAD), venous stasis, transplant history with immunosuppression, hepatitis C, HIV, rheumatoid arthritis, body mass index, hemoglobin A1C, anatomical wound location, weightbearing status of the wound, wound size at the time of placement of the STSG, wound size 30 days post-STSG, wound size 60 days post-STSG, number of debridements prior to STSG placement, number of prior STSGs, STSG thickness, type of wound bed preparation (Integra, EZ-Derm Porcine Xenograft, or none), type of wound dressing (bolster or NPWT), species and amount of bacteria for post-debridement cultures during the visit prior to the operation, species and amount of bacteria for pre-debridement cultures just prior to STSG placement, species and amount of bacteria for post-debridement cultures just prior to STSG placement, percent take of the STSG 30 and 60 days post STSG placement, presence of infection 30 and 60 days post-STSG placement, presence of hematoma 30 and 60 days post-STSG placement, presence of seroma 30 and 60 days post-STSG placement, and general outcome after STSG placement. Inclusion of data for each of these components was limited only by the presence of data and patient compliance to 30 and 60 day follow-up appointments (Table 1 and 2). These elements were recorded for each wound, and thus patients with multiple distinct wounds had multiple entries based on each distinct wound. Further analyses may be warranted to ensure that the presence of concomitant wounds does not affect the efficacy of the healing process; however, due to the low prevalence of patients with multiple wounds it is unlikely to have significantly affected the results of this particular study.

\section{Statistical Analysis:}

Continuous variables were described by means and standard deviations, and two sample t-tests were used to compare distribution of continuous variables. Categorical variables were described by frequencies and percentages, and Chisquare and Fisher's exact tests were used as appropriate to compare proportions of categorical variables. Multivariate logistical regression analysis was performed to determine the stated factors that affect the outcome of STSG, adjusting for variables which are found significant in the bivariate analysis (Table 3). Odds ratio of the statistically significant factors were taken to determine the degree of correlation between poor outcome and the given factor. Outcome of STSG was determined to be either successful or not based on an $80 \%$ take of the STSG. STSG take was based on percent change in initial wound size recorded or if explicitly stated in the patient charts at the 30 and 60 day post-operative appointment. Statistical significance was defined as $\mathrm{p}<0.05$. 


$\begin{array}{ll}\text { Characteristic } & \\ \text { Gender } & \\ \quad \text { Female } & 72(37.7 \%) \\ \quad \text { Male } & 119(62.3 \%) \\ \text { Average Age (years) } & 59.8 \pm 14.3 \\ \text { Average Body Mass Index }\left(\mathrm{kg} / \mathrm{m}^{2}\right) & 31.6 \pm 8.4 \\ \text { Diabetes Mellitus } & 123(64.4 \%) \\ \text { Average Hemoglobin A1C }(\%) & 6.85 \pm 1.8 \\ \text { Hypertension } & 159(83.3 \%) \\ \text { Hyperlipidemia } & 109(57.1 \%) \\ \text { Congestive Heart Failure } & 38(19.9 \%) \\ \text { Renal Failure } & 63(33.0 \%) \\ \text { Peripheral Artery Disease } & 82(42.9 \%) \\ \text { Venous Stasis } & 39(20.4 \%) \\ \text { Transplant Suppression } & 7(3.7 \%) \\ \text { Hepatitis C } & 14(7.3 \%) \\ \text { Human Immunodeficiency Virus } & 7(3.7 \%) \\ \text { Rheumatoid arthritis } & 10(5.2 \%) \\ \text { Patients with Multiple Concomitant Wounds } & \\ \quad 2 \text { Wounds } & 19(9.9 \%) \\ \quad 3 \text { Wounds } & 5(2.6 \%)\end{array}$

\section{Results}

STSGs were placed for 223 lower extremity wounds involving 191 patients with an average age of $59.8 \pm 14.3$ and an average BMI of $31.6 \pm 8.4$. The population contained more male $(62.3 \%)$ than female patients $(37.7 \%)$. The average wound size was $73.25 \mathrm{~cm}^{2}$ with an average take of $70.3 \% 30$ days post-operative and $80.9 \% 60$ day post-operative and a total of $31.8 \%$ rate of failure of all STSGs in the patient population. The correlation of each of the components reviewed in the patient records was analyzed to determine its effect on skin graft take, and thus its success or failure. Of the components reviewed in the patient's chart, only CHF, bacterial presence, and post-procedural dressing type were significant factors that had a direct effect on STSG outcome.

Patients who had NPWT after STSG placement constitute a significantly higher proportion of the STSG success population compared to the population that garnered STSG failure $\left[\mathrm{n}_{\mathrm{s}}=78(51.7 \%)\right.$ versus $\mathrm{n}_{\mathrm{f}}=26(36.6 \%)$, $(\mathrm{p}=0.0363)]$. Further statistical analysis showed a significant association between NPWT and STSG success compared to bolster use only $\left(\chi^{2}=4.66\right.$, $\mathrm{p}=0.0308$ ), where the odds of STSG failure in patients who underwent NPWT were approximately $80 \%$ less than those who had bolster dressing only $(\mathrm{OR}=$ 0.203 ). 


\begin{tabular}{|c|c|}
\hline \multicolumn{2}{|l|}{ Characteristic } \\
\hline Average Initial Wound Size & $72.9 \mathrm{~cm}^{2}$ \\
\hline \multicolumn{2}{|l|}{ Wound Location } \\
\hline Foot & $106(47.5 \%)$ \\
\hline Leg & $117(52.5 \%)$ \\
\hline Weightbearing Surface & $50(22.1 \%)$ \\
\hline \multicolumn{2}{|l|}{ Most Recent Wound Bed Preparation } \\
\hline STSG alone & $52(23 \%)$ \\
\hline EZ-Derm & $35(15 \%)$ \\
\hline Integra & $137(606.6 \%)$ \\
\hline \multicolumn{2}{|l|}{ Wound Dressing } \\
\hline NPWT & $106(47.1 \%)$ \\
\hline Bolster & $119(53.1 \%)$ \\
\hline \multicolumn{2}{|l|}{ Previous STSGs } \\
\hline 0 & $189(83.6 \%)$ \\
\hline 1 & $25(11.1 \%)$ \\
\hline 2 & $6(2.7 \%)$ \\
\hline 3 & $2(0.9 \%)$ \\
\hline 4 & $4(1.8 \%)$ \\
\hline Average Wound Size 30 days Post-op & $10.4 \mathrm{~cm}^{2}$ \\
\hline Average Wound Size 60 days Post-op & $6.0 \mathrm{~cm}^{2}$ \\
\hline \multicolumn{2}{|l|}{ Complications 30 days Post-op } \\
\hline Infection & $5(2.2 \%)$ \\
\hline Hematoma & $3(1.3 \%)$ \\
\hline Seroma & $0(0 \%)$ \\
\hline \multicolumn{2}{|l|}{ Complications 60 days Post-op } \\
\hline Infection & $9(3.98 \%)$ \\
\hline Hematoma & $1(0.4 \%)$ \\
\hline Seroma & $1(0.4 \%)$ \\
\hline
\end{tabular}

Patients with CHF constitute a significantly higher proportion of the STSG failure population compared to the population that garnered STSG success $\left[\mathrm{n}_{\mathrm{f}}=19(26.8 \%)\right.$ versus $\left.\mathrm{n}_{\mathrm{s}}=23(15.1 \%),(\mathrm{p}=0.0385)\right]$. Further statistical analysis showed a significant association between CHF and STSG failure $(\chi$ $2=4.12, p=0.0422)$ where patients with CHF were approximately 2.55 times more likely to have STSG failure $(\mathrm{OR}=2.55)$.

Patients with bacterial presence before the debridement done just prior to STSG placement constitute a significantly higher proportion of the STSG failure population compared to the population that garnered STSG success $\left[\mathrm{n}_{\mathrm{f}}\right.$ $=58(81.7 \%)$ versus $\left.n_{s}=98(64.5 \%),(p=0.0090)\right]$. Further statistical analysis showed a significant association between this bacterial presence and STSG failure $\left(\chi^{2}=4.66, \mathrm{p}=0.0308\right)$ where patients with bacterial presence before the debridement were approximately 2.89 times more likely to have STSG failure $(\mathrm{OR}=2.89)$. Although bacterial presence prior to debridement showed an 
Fully healed wounds (>80\% take) $n=152$

Demographics and Medical History

Age

Gender

Female

Male

Body Mass Index

Diabetes Mellitus

Hemoglobin A1C

Hypertension

Hyperlipidemia

Congestive Heart Failure

Renal Failure

Transplant Suppression ${ }^{1}$

Hepatitis $C^{1}$

Human Immunodeficiency Virus

Rheumatoid arthritis

Peripheral Arterial Disease

Venous Stasis

Wound Parameters

Wound Location

$$
\text { Foot }
$$

Leg

Weightbearing status

Non-weightbearing

Weightbearing

Number of previous DBT

Number of previous STSGs

$$
0
$$

1

2

3

4

Most Recent Wound Bed Preparation

STSG alone

EZ-Derm

Integra

Wound Dressing

NPWT

Bolster

Wound Microbiology

Presence of Bacteria on Culture

Post-debridement culture in OR visit prior to STSG placement

$76(50.0 \%)$

$98(64.5 \%)$

$95(62.5 \%)$

$58.8 \pm 14.2$

$86(38.1 \%)$

$140(61.9 \%)$

$100(65.2 \%)$

$6.8 \pm 1.8$

$126(82.9 \%)$

$93(61.2 \%)$

$23(15.1 \%)$

$48(31.6 \%)$

$1(0.7 \%)$

6 (3.95\%)

$9(5.9 \%)$

6 (3.95\%)

$63(41.5 \%)$

$30(19.7 \%)$

$69(45.4 \%)$

$73(48.0)$

STSG placement
Unhealed wounds $(<80 \%$ take) $n=71$

value

$60.5 \pm 14.0$

0.4066

$24(33.3 \%)$

$47(66.7 \%)$

$32.3 \pm 8.9$

0.2642

$50(70.4 \%)$

0.4922

$7.3 \pm 2.4$

0.2006

$64(90.1 \%)$

0.1557

$37(52.1 \%)$

0.2006

$19(26.8 \%)$

0.0385

30 (42.3\%)

0.1194

$6(8.5 \%)$

0.0047

$10(14.1 \%)$

0.0063

$4(5.6 \%)$

0.9320

$4(5.6 \%)$

0.7294

$36(50.7 \%)$

0.1949

$20(28.2 \%)$

0.1596

0.3494

37 (52.1\%)

34 (47.9\%)

0.2390

52 (73.2\%)

19 (26.8\%)

$3.5 \pm 1.7$

0.3664

0.5840

$62(87.3 \%)$

7 (9.9\%)

1 (1.4\%)

1 (1.4\%)

0 (0\%)

1.0000

16 (22.5\%)

11 (15.5\%)

44 (62.0\%)

0.0363

26(36.6)

45(63.4)

$39(54.9 \%)$

0.4926

$58(81.7 \%)$

0.0090

52 (73.2\%)

0.1150 
Fully healed wounds $(>80 \%$

take) $n=152$
Unhealed wounds $(<80 \%$

take) $n=71$ p-

value

Demographics and Medical History

\section{STSG placement}

Post-operative Complications

Complications at the 30 -day post-operative visit

Infection

Hematoma

$1(0.7 \%)$

$2(2.8 \%)$

0.2401

Seroma

$0(0.0 \%)$

$0(0.0 \%)$

1.0000

Complications at the 60-day post-operative visit

Infection

Hematoma

1-Although statistically significant these factors were not found to be significant with multivariate analysis

association with STSG failure, bacterial presence after debridement just prior to STSG placement did not show a significant correlation with STSG failure $\left[n_{\mathrm{f}}=52(73.2 \%)\right.$ versus $\left.n_{\mathrm{s}}=95(62.5 \%),(\mathrm{p}=0.1150)\right]$.

\section{Discussion NPWT}

NPWT is a recent discovery in wound healing that has been introduced as a wound dressing as an alternative to conventional compressive bolster dressings (CBDs). Because of its promising effects in decreasing surgical site complications, extensive research has been done on its efficacy compared to $\mathrm{CBD}$ to see whether these effects have any basis. On the basis of animal studies, it is posited that NPWT is successful in wound therapy due to its ability to promote cell proliferation, angiogenesis, and capillary blood flow, allowing for deliverance of oxygen, nutrients, and healing factors that would allow for quicker and more reliable healing, 2,3 . A human study by Kamolz et al. on the effects of NPWT the progression of burns also showed hyperperfusion of the site, as well as edema reduction. ${ }^{4}$ Negative pressure not only allows the NPWT to pull out fluid, leading to reduction in edema and hematoma formation, but also allows for uniform compression, minimizing shear stress. Edema reduction leads to further increased oxygen and nutrient delivery to tissues, leading to increased angiogenesis and granulation tissue formation, as well as removal of inhibitory factors from chronic inflammation. In addition, clinical studies have shown its efficacy in post-surgical wound therapy. Multiple meta-analyses analyzing the outcomes of post-surgical wounds dressed with NPWT compared to CBDs found significantly reduced not only incidence of post-surgical complications, such infection, seroma, and wound dehiscence, 
but length of stay, as well. ${ }^{5,6}$ Despite this extensive research that indicates a crucial benefit of NPWT in post-surgical wounds, the clinical significance for NPWT over CDB in skin grafts has not been well established. ${ }^{7}$

Based on our results we found a significant association between NPWT and STSG success compared to CBD, where the odds of STSG failure dropped approximately $80 \%$ with use of NPWT over CBD. In a similar retrospective study, Scherer et al. found that significantly more STSGs with CBD failed; however, their data was skewed due to significantly larger wounds in the CBD group. ${ }^{8}$ In our study, the risk of STSG failure was decreased despite a larger average wound size dressed with NPWT $\left(85.77 \mathrm{~cm}^{2}\right)$ compared to CBD $(60.64$ $\mathrm{cm}^{2}$ ). Another similar retrospective study performed by Kempton et al. showed no significant difference between NPWT and CBD in the success of STSG take, and based on this data and a cost analysis concluded that NPWT is not a viable alternative to CBD; however, the patients included in this study were treated only for low risk wounds with a low STSG failure rate, and the cost analysis did not account for the monetary expense of an extended stay in the hospital needed for CBD. ${ }^{9}$ From our analysis, NPWT has a significant benefit over CBD, reducing risk of STSG failure in a wide range of wound types and sizes, and thus has both the statistical and clinical significance necessary to be deemed appropriate to use in the case of most wounds over bolster dressing. A prospective, randomized study on the effect of NPWT versus $\mathrm{CBD}$ on wounds with varying levels of acuity from low to high risk of STSG failure could be helpful in determining a distinct guideline for when it is most appropriate to use NPWT.

\section{Congestive Heart Failure}

Comorbidities, such as DM and peripheral vascular disease, have been shown to play a pivotal role in the impairment of healing processes that lead to graft failure, however little research has been published on the effects of CHF on skin graft take. There is a dearth of published data that has detailed the significance of CHF in STSG outcomes, however our data showed that patients with CHF had a 2.55 time higher risk for STSG failure. In a similar retrospective study, Rhou et al. analyzed the comorbidities that were predictive of poor overall healing in diabetic foot ulcers. They found that only $65 \%$ of ulcers in patients with CHF healed within 12 weeks, whereas $91 \%$ of ulcers in patients without CHF healed within 21 weeks, and those that healed in patients with CHF had a slower healing rate. The presence of CHF was found to be the only factor independently predictive of failure to heal overall, delayed healing at 12 weeks, and reduced rate of healing per week. ${ }^{10}$ Skin grafts require proper adherence and revascularization to properly take and heal a wound, so the same factors that are detrimental to wound healing would be equally as detrimental to skin graft take. The effect of CHF on wound healing, and thus skin graft take, is likely multi-factorial. Two likely factors that may contribute to CHF's effect on healing are tissue ischemia and edema. Because the heart is unable to pump efficiently, CHF is associated with tissue ischemia and edema. 
If blood is unable to reach the tissue to supply oxygen, nutrients, and proper healing and angiogenic factors, it will not reach the graft, as well, and the graft will fail, while edema may provide a further barrier for oxygen diffusion across the capillaries and reduce the clearance of metabolites, which may cause further tissue damage or impair healing and graft take. ${ }^{11}$ Edema also affects adherence by providing a poor environment for granulation tissue formation, as well as increasing shearing forces and providing a poor landscape for adhesion, similar to hematoma or seroma formation. The success of compressive dressing, whether it is via bolster or NPWT, points to edema as a factor in poor wound healing. ${ }^{11}$ Tissue ischemia and edema are, however, common in other comorbidities, so further prospective analysis should be performed to determine why CHF is a successful independent predictor of wound healing and graft take.

\section{Bacterial presence}

Nearly all chronic wounds contain bacteria. The presence of bacteria can range from contamination to infection; however, the effect of bacteria depends on the amount and strain of bacteria, as well as the patient's ability to mount an immunological response. In patients with dysfunctional immune systems, even normal skin flora can yield chronic wound infection. ${ }^{12}$ Chronic wounds are generally caused by a persistent, inflammatory state, which can be perpetuated by persistent infection. Chronic inflammation is often characterized by high amounts of polymorphonuclear leukocytes (PMNL), macrophages, and lymphocytes at the wound bed, as well as an imbalance of inflammatory cytokines, which causes an imbalance of synthesis and degradation of the extracellular matrix (ECM). A study by Amato et al. showed that overexpression of metalloproteinases, which regulate the ECM and its remodeling during wound repair, led to the prevention of normal ECM formation and remodeling, and thus a non-healing wound. ${ }^{13}$ This inflammatory process that causes tissue destruction is further exacerbated by the presence of bacteria, particularly highly immunogenic bacteria that has colonized, which activates the innate immune response for further infiltration of PMNLs and production of inflammatory cytokines. The most common bacteria found in pre-operative swabs for our study was Coagulase negative Staphylococci, followed by Methicillin-Resistant Staphylococcus Aureus, Diptheroids, Staphylococcus Aureus, and Psuedomonas Aeruginosa respectively.

Studies done by Hogsberg et al. and Gilliland et al. showed that specific strains, particularly Pseudomonas Aeruginosa and Staphylococcus Aureus had a deleterious effect on STSG outcomes. ${ }^{14,15}$ Further analysis of the data assessing the comparative effects of each bacterial strain on STSG failure is necessary. It is also important to determine whether a wound bed site that is nearing sterility post-debridement is necessary for successful take of the STSG. A study performed by Aerden et al. found that of the 87 wounds that they had analyzed, those that were near sterile did not have an improved mean graft take compared to wounds that were contaminated, having $87 \%$ and $90 \%$ 
mean take respectively. This study also found a worse outcome for STSG take for Pseudomonas Aeruginosa and Staphylococcus Aureus. Because of these results, Aerden et al. concluded that identification of the strain of bacteria via qualitative analysis of the wound culture via pre-operative swabs was more important than quantitative analysis to determine efficacy of STSG take. ${ }^{16}$ Similarly, our data showed no association between bacterial presence just prior to STSG placement and STSG failure; however, without establishment of a control group, the magnitude of the effect in outcome in our study cannot be made. Further quantitative and qualitative analysis of bacterial amount and type of bacteria and they effect on wound healing would be required in order to truly elucidate the effect of bacteria on STSG take; however, the fact that bacterial presence was found to not have an effect on the success STSG placement is still an important finding.

\section{Conclusions}

Negative pressure wound therapy application after skin graft placement yields a greater success rate for split-thickness skin grafts than conventional bolster dressing. In addition, congestive heart failure is a good predictor of splitthickness skin graft failure. Bacterial presence in culture may also be a good predictor of graft failure, however it is possibly the strain of bacteria, not the presence of bacteria that predominantly affects skin graft take. Further data analysis is warranted. 


\section{REFERENCES}

1. Llanos S, Danilla S, Barraza C, et al. Effectiveness of negative pressure closure in the integration of split thickness skin grafts: a randomized, double-masked, controlled trial. Annals of surgery. 2006;244(5):700-705.

2. Scherer SS, Pietramaggiori G, Mathews JC, et al. The mechanism of action of the vacuumassisted closure device. Plast Reconstr Surg. 2008;122:786-797.

3. Chen SZ, Li J, Li XY, et al. Effects of vacuum-assisted closure on wound microcirculation: an experimental study. Asian J Surg. 2005;28:211-217.

4. Kamolz LP, Andel H, Haslik W. Use of subatmospheric pressure therapy to prevent burn wound progression in human: first experiences. Burns. 2004;30:253-258.

5. Strugala V, Martin R. Meta-Analysis of Comparative Trials Evaluating a Prophylactic Single-Use Negative Pressure Wound Therapy System for the Prevention of Surgical Site Complications. Surgical Infections. 2017;18(7):810-819.

6. Hyldig N, Birke-Sorensen H, Kruse M, et al. Meta-analysis of negative-pressure wound therapy for closed surgical incisions. The British Journal of Surgery. 2016;103(5):477-486.

7. Webster J, Scuffham P, Stankiewicz M, Chaboyer WP. Negative pressure wound therapy for skin grafts and surgical wounds healing by primary intention. Cochrane Database Syst Rev. 2014.

8. Scherer LA, Shiver S, Chang M, et al. The vacuum assisted closure device: a method of securing skin grafts and improving graft survival. Arch Surg. 2002;137:933-934.

9. Kempton LB, Larson TB, Montijo HE, et al. Increased cost of negative pressure dressings is not justified for split thickness skin grafting of low-risk wounds.J Orthop Trauma. 2015;29:301-306.

10. Rhou YJ, Henshaw FR, McGill MJ, Twigg SM. Congestive heart failure presence predicts delayed healing of foot ulcers in diabetes: an audit from a multidisciplinary high-risk foot clinic. $J$ Diabetes Complic. 2015;29(4):556-562.

11. Mustoe TA, O’Shaughnessy K, Kloeters O, et al. Chronic wound pathogenesis and current treatment strategies: A unifying hypothesis. Plastic and Reconstructive Surgery. 2006;117:35S-41S.

12. Ugur A, Ceylan O. Occurrence of resistance to antibiotics, metals, and plasmids in clinical strains of Staphylococcus spp. Arch Med Res. 2003;34:130-136.

13. Amato B, Coretti G, Compagna R, et al. Role of matrix metalloproteinases in non-healing venous ulcers. Int Wound J. 2013:1-5.

14. Høgsberg T, Bjarnsholt T, Thomsen JS, Kirketerp-Møller K. Success rate of split-thickness skin grafting of chronic venous leg ulcers depends on the presence of Pseudomonas aeruginosa: a retrospective study. PLoS One. 2011;6(5).

15. Gilliland EL, Nathwani N, Dore CJ, Lewis JD. Bacterial colonisation of leg ulcers and its effect on the success rate of skin grafting. Ann R Coll Surg Engl. 1988;70:105-108.

16. Aerden D, Bosmans I, Vanmierlo B, Spinnael J, Keymeule B, Van den Brande P. Skin grafting the contaminated wound bed: reassessing the role of the preoperative swab.J Wound Care. 2013;22:85-89. 